\title{
Cross-listed Securities and Multiple Exchange Memberships: Demand Differentiability and Equilibrium Existence
}

\author{
${ }^{1}$ Universidade Nova de Lisboa, FCT and CMA, Lisbon, Portugal, E-mail: mcm@fct.unl.pt \\ ${ }^{2}$ University of Wisconsin - Madison, 5259 Grainger Hall, 975 University Avenue, Madison, WI 53706, USA, E-mail: \\ jluque@bus.wisc.edu
}

\begin{abstract}
:
The previous literature on general equilibrium has assumed that all traders belong to a single market. However, traders often participate in more than one exchange to diversify their portfolios. Moreover, there is evidence that the security listings of exchanges overlap. Our model captures these facts: there are multiple exchanges, the same security is listed in different exchanges, and traders can belong to more than one exchange. We show that, in the presence of convex transaction costs, there exists an equilibrium, and that individual demand is a continuously differentiable function in prices.
\end{abstract}

Keywords: multiple exchange memberships, cross-listings, equilibrium existence, demand differentiability JEL classification: D52, D53, G12, G14, G15, G18

DOI: 10.1515/bejte-2016-0114

\section{Introduction}

During the last few decades, the securities exchange industry has experienced an intense demutualization process. For example, in 2007 the European Commission enacted the Market in Financial Instruments Directive (Mifid) to facilitate competition across the region. Similarly, the U.S. has encouraged fragmentation with the Regulation National Market System (see the 2010 SEC report "Concept Release on Equity Market Structure"). The result has been a more interconnected market structure with "overlapping security exchanges", where traders participate in several exchanges (multiple memberships), and securities are simultaneously traded in different platforms (cross-listings).

Several empirical studies have echoed the importance of cross-listings. For instance, Krishnamurti, Sequeira, and Fangjian (2003) have documented 40 stocks that are simultaneously traded on two Indian stock exchanges. Treptow (2006) presented evidence that the number of cross-listed securities between the NYSE and the respective primary market is quite significant: Amsterdam (20), Athens (5), Australia (11), Brussels (1), Deutsche Bourse (16), Helsinki (4), Hong Kong (9), Lisbon (2), London (53), New Zealand (2), Oslo (4), Paris (21), Singapore (1), Stockholm (1), Toronto (58), where the number in parentheses stands for the number of cross-listings.

We incorporate these new features into the standard and simplified setting of general financial equilibrium models with a single consumption good, several securities, and two dates. Traders are small and take prices as given. ${ }^{1}$ At date 1 traders buy and sell securities in their respective exchanges, subject to transaction costs. We do not explain at all how traders have chosen their exchange memberships. Instead, memberships enter as a primitive in our formulation. ${ }^{2}$ At date 2 there are several states of nature where real securities pay exogenous returns.

Multiple memberships and cross-listings introduce two new fundamental challenges in the classic twoperiod financial general equilibrium model. One has to do with demand differentiability and the other with equilibrium existence. We find positive results for both cases when trading costs are convex. This convexity property of trading costs is motivated by some frictions that a trader faces when buying/selling a security. For example, we have in mind search costs, i.e., the larger the quantity bought (sold), the more difficult it is for a trader to find the security (the buyer). Another example is the costs associated with the valuation of a security, e.g., the larger the amount of a mortgage-backed security (MBS) bought, the more due diligence the buyer needs to do in order to properly understand and value the pool of mortgages backing the MBS.

In the remainder of this Introduction, we explain our two main results separately. 


\subsection{Demand Differentiability}

Cross-listings prevent us from using previous techniques to prove the differentiability of the demand function with respect to prices (Geanakoplos and Polemarchakis 1986 assumed that the asset payoff matrix has full rank): if a trader belongs to two exchanges and the same security is listed in these two exchanges, this trader's available security payoff structure becomes linearly dependent. Thus, this trader's security payoff structure may not have full column rank. We refer to this possibility as the "collinearity problem". ${ }^{3}$ A (non-linear) convex transaction cost guarantees that traders' demand functions are smooth in a context with collinear securities among different exchanges (for this result, trading costs do not need to be large). Key for this result is to show that the Jacobian matrix of the system of first order conditions has full column rank even when the trader's matrix of security returns does not have full rank. This property may fail when we consider instead a concave trading cost function.

The (positive) result of the smoothness of the demand function can be useful not only for standard analytical exercises, but also to study other important theoretical issues, such as the regularity of the competitive equilibrium or the endogeneity of an exchange structure with multiple memberships and cross-listings. For the latter, Faias and Luque (2017) show that demand differentiability is crucial in evaluating the trader's utility function in a given trading equilibrium for a given exchange structure. However, the approach of endogenizing the exchange structure in Faias and Luque (2017) is club theory, and such a framework assumes that a trader belongs to only one exchange. Allowing for multiple memberships would imply that the trader's utility depends on the whole structure of exchanges through prices, a setting incompatible with existing results in club theory. Thus, it remains an open question how to endogenize the structure of security exchanges with multiple memberships. The results in the current paper make a step towards that goal by identifying conditions under which demand differentiability is achieved.

\subsection{Equilibrium Existence}

The standard simultaneous optimization approach to equilibrium existence of Debreu (1952) and Arrow and Debreu (1954), which relies on fixed point theory, fails when considering more than one platform (exchange). In that setting, there is an auctioneer that chooses both the commodity and security prices in the simplex. If the auctioneer chooses the price of one security equal to 1 , the remaining commodity and security prices would be zero. But then, there would be exchanges with commodity and security prices equal to zero, and the budget constraints of traders with single exchange memberships would hold with equality. In that case, we cannot guarantee the lower semicontinuity property of the budget correspondence by finding an interior point in the budget constraint.

Another tentative approach would be to consider an auxiliary economy for each exchange where traders trade the consumption good and securities only among themselves, and then show that the equilibrium for this system of auxiliary economies is a trading equilibrium for the original economy. However, with multiple memberships, we would not be able to isolate the trading activity in one exchange from the trading in another exchange.

We are able to show existence of equilibrium for our model with cross-listings, multiple memberships, and market clearing occurring in each exchange using fixed point theorem. A subtlety of our existence proof is guaranteeing the lower semicontinuity of the trader's budget set correspondence. For this, we need to show that exchange clearing security prices cannot be "too large" in equilibrium. To this end, we cannot impose additional restrictions on the trader's budget constraints and portfolio sets, as these are written to capture our particular setting. We do not require further assumptions concerning portfolio sets, namely, "financial survival" assumptions as in Angeloni and Cornet (2006) and Aouani and Cornet (2009, 2011), which would not be in accordance with our framework. Instead, we consider a mild impatience assumption, proposed by Seghir and Torres-Martinez (2011) for an economy with restricted participation. This assumption, satisfied by many types of utility functions (e.g., CES, CARA, Cobb-Douglas), says that the impact on the welfare of a reduction in future consumption can be offset by an increase in today's consumption. ${ }^{4}$ The assumption does not depend on the representation of individuals' preferences.

Another subtlety of our existence proof is the presence of transactions costs, which are paid in the numeraire good. They allow us to obtain endogenous bounds on the trader's portfolio choice set. This approach differs from what has been done in the literature of incomplete financial markets with exogenous portfolio constraints. Specifically, we do not need to consider an auxiliary economy where the original financial economy (the financial structure or the set of admissible portfolio allocations) is reduced without changing the consumption equilibrium set (see Angeloni and Cornet 2006; Aouani and Cornet 2009; Balasko, Cass, and Siconolfi 1990; Cornet and Gopalan 2010, among others).

For our existence proof, it is convenient to assume that transaction costs are convex because they do not change the convex nature of the values of the budget constraint correspondence. Equilibrium existence with 
concave transaction costs could be recovered if we extend our model to a large economy with a continuum of traders.

\subsection{Structure of the Paper}

The remainder of this paper is structured as follows. Section 2 establishes the baseline model. Section 3 establishes the equilibrium existence and demand differentiability results. Here, we also provide further discussion of the contribution of our paper to the literature of restricted participation. In Section 4, we address important issues regarding our model. First, we provide an example that illustrates how the equilibrium in a setting with decentralized (multiple) exchanges differs from its counterpart in a setting with a (unique) centralized exchange. Second, we discuss the economic interpretation and plausibility of convex transaction costs and the implications of considering instead a concave transaction cost function, both in terms of equilibrium existence and demand differentiability. Third, we show that our baseline model is a particular case of a more general model that only considers a single global financial market and restrictions on the trader's portfolios. Also there we explain that previous results obtained in the literature of restricted participation cannot be applied to show that an equilibrium exists for our more general model. Section 5 concludes and suggests directions for future research.

\section{The Baseline Model}

An exchange participant or trader is a corporation that may trade on or through the exchange and is licensed under the ordinance of the corresponding exchange financial regulator to carry out security trading activity. An exchange is a security market that allows traders with membership in that exchange to diversify risk among themselves by trading the securities available in the exchange.

\subsection{Uncertainty, Trader's Primitives, and Security Payoffs}

Our economy lasts for two dates, $t=1,2$. The set of states of uncertainty in the last date is $\Xi \equiv\{1, \ldots, \Xi\}$, with representative element $\xi$. At each date there is a spot market for a perfectly divisible perishable physical good, whose price we normalize to 1 . Thus, the commodity space is $\mathrm{IR}_{+}^{\Xi+1}$. For a consumption bundle, we use the notation $x=\left(x_{1},(x(\xi), \xi=1, \ldots, \Xi)\right) \in \mathrm{IR}_{+}^{\Xi+1}$, where $x_{1}$ denotes the consumption at the first date and $x(\xi)$ denotes the consumption at state $\xi$ of the second date.

The set of traders is $\mathbf{I} \equiv\{1, \ldots, I\}$, with $I$ assumed to be large but finite. We write $x^{I}=\left(x^{i}: i \in \mathbf{I}\right)$ to denote traders' consumption bundles in the two dates. Each trader is endowed with a consumption set $X^{i}=\operatorname{IR}_{+}^{\Xi+1}$, a utility function $u^{i}: X^{i} \rightarrow \mathrm{IR}$, and an endowment vector $\omega^{i}=\left(\omega_{1}^{i},\left(\omega^{i}(\xi), \xi=1, \ldots, \Xi\right)\right) \in \mathrm{IR}_{++}^{\Xi+1}$.

At date 1, security trading occurs in several exchanges. At date 2, these securities pay returns. The set of securities in the economy is denoted by $\mathbf{J} \equiv\{1, . ., J\}$. We denote security $j^{\prime}$ s return (in terms of the good) at state $\xi$ by $a_{j}(\xi), a_{j}(\xi) \geq 0$.

\subsection{Exchange Structure and Trading}

By an exchange $E$ we mean a group of traders $(S)$ and a payoff matrix associated with the subset of securities $J(E) \subseteq \mathbf{J}$ available for trade in an exchange $E$. We denote this payoff matrix by $A=\left[a_{j(E)}(\xi)\right]_{\Xi \times J(E)}$. Thus, we write $E=(S, A)$ for an exchange in general. Let us then denote an exchange structure by $F(\mathbf{I})=\left\{E_{k}\right\}_{k=1}^{K}=$ $\left\{\left(S_{k}, A_{k}\right)\right\}_{k=1}^{K}$ (notice that because we allow for multiple exchange memberships, the set $\left\{S_{k}\right\}_{k=1}^{K}$ is not a partition of I). A key element of our model is that traders can only trade securities in those exchanges to which they belong. Formally, we denote by $F[i ; \mathbf{I}]=\left\{E_{k} \in F(\mathbf{I}): i \in S_{k}\right\}$ the set of all exchanges in $F$ (I) that contain trader $i$.

We denote trader $i$ 's trading of security $j(E) \in J(E)$ at date 1 by $y_{j(E)}^{i}$. A necessary condition for trader $i$ to trade $j(E)$ is that $(S, A) \in F[i ; \mathbf{I}]$. As usual, $y_{j(E)}^{i}>0$ denotes a purchase of security $j(E)$, while $y_{j(E)}^{i}<0$ denotes a sale of this security. We write $y^{I}=\left(y^{i} \in \mathrm{IR}^{J\left(E_{k}\right)}: E_{k} \in F[i ; I], i \in I\right)$ to denote the vector of security positions that all traders have in their respective exchanges. 
Traders face transaction costs $g_{j(E)}\left(y_{j(E)}\right)$ when trading an amount $y_{j(E)}$ of security $j(E)$. Transaction costs are denominated in units of the private good, and apply to both sides (long and short security positions). For simplicity, we assume that these costs do not revert to the individuals of the economy. ${ }^{5}$ Given the exchange structure $F(\mathbf{I})$ and security prices $q \in I R^{\sum_{k=1}^{K}\left|J\left(E_{k}\right)\right|}$, trader $i^{\prime}$ s budget constraint at date 1 is

$$
x_{1}-\omega_{1}^{i}+\sum_{E_{k} \in F[i ; \mathbf{I}]} \sum_{j\left(E_{k}\right) \in J\left(E_{k}\right)}\left(q_{j\left(E_{k}\right)} y_{j\left(E_{k}\right)}+g_{j\left(E_{k}\right)}\left(y_{j\left(E_{k}\right)}\right)\right) \leq 0 .
$$

Trader i's budget constraint at date 2 and node $\xi \in \Xi$ is such that his consumption, net of his good endowment, is bounded by the returns on his security positions.

$$
x(\xi)-\omega^{i}(\xi) \leq \sum_{E_{k} \in F[i ; \mathbf{I}]} \sum_{j\left(E_{k}\right) \in J\left(E_{k}\right)} a_{j\left(E_{k}\right)}(\xi) y_{j\left(E_{k}\right)} .
$$

\subsection{Assumptions}

We impose the following assumption on transaction costs:

(A1) $g_{j\left(E_{k}\right)}: I R \rightarrow I R_{+}$is twice continuous differentiable $\left(C^{2}\right)$ in $y_{j\left(E_{k}\right)}$, increasing in $\left|y_{j\left(E_{k}\right)}\right|$, convex in $y_{j\left(E_{k}\right)}$ (i.e., $\left.g^{\prime \prime}>0\right)$, and is such that $g(0)=0$, for all $j\left(E_{k}\right) \in J\left(E_{k}\right)$ and all $E_{k} \in F[\mathbf{I}]$.

We need the properties in (A1) for both our equilibrium existence and demand differentiability results. We refer to Section 4.2 for the economic interpretation of the convexity of the transaction cost function.

Trader $i^{\prime}$ s utility function $u^{i}: \mathbb{R}_{+}^{1+\Xi} \rightarrow \mathbb{R}$, evaluated on the consumption bundle $x \in \mathbb{R}_{+}^{1+\Xi}$, is denoted by $u^{i}\left(x_{1}, x(1), \ldots, x(\Xi)\right)$, and satisfies the following assumptions:

(A2) For every $i \in \mathbf{I}, u^{i}$ is continuous, strictly quasi-concave, and strictly increasing on $I R_{+}^{1+\Xi}$.

(A3) For every $i \in \mathbf{I}, u^{i}$ is twice continuously differentiable and the matrix of second derivatives, $D^{2} u^{i}$, is negative definite on $I R_{++}^{1+\Xi}$.

Assumptions (A2) and (A3) are standard (see Geanakoplos and Polemarchakis 1986). We also consider utility functions that satisfy a mild impatience assumption that says that we can always find a large consumption at date 1 such that the trader is better off with this extra consumption at date 1 but less consumption in every state of date 2. Formally, we write this as follows:

(A4) For every $i \in \mathbf{I}$, given a consumption plan $x=\left(x_{1}, x(1), \ldots, x(\Xi)\right) \in I R_{++}^{1+\Xi}$, for any $\theta \in(0,1)$ there exists a bundle $\varrho(\theta, x) \in I R_{+}$, such that $u^{i}\left(x_{1}+\varrho(\theta, x), \theta x(1), \ldots, \theta x(\Xi)\right)>u^{i}(x)$.

As Seghir and Torres-Martinez (2011) point out, this assumption is satisfied by many different types of utility functions. For instance, it is satisfied by utility functions that are unbounded on the first date consumption, such as von-Neumann utility functions with quasi linear, Cobb-Douglas, or Leontieff kernels (e.g., Cobb-Douglas, CES, CARA). Also notice that this type of utility function does not depend on the representation of individuals' preferences and does not require further assumptions on the portfolio sets.

\section{Existence of Equilibrium and Demand Differentiability}

In this section we give our two main results: equilibrium existence and demand differentiability. We start with the former.

\subsection{Existence of Equilibrium}

Given an exchange structure $F(\mathbf{I})$, an equilibrium consists of a system $\left(x_{1}^{I}, x^{I}(1), \ldots, x^{I}(\Xi), y^{I}, q\right) \in I R_{+}^{I(1+\Xi)} \times$ $I R^{\sum_{i \in \mathbf{I}} \sum_{E_{k} \in F[i ; \mathbf{I}]}\left|J\left(E_{k}\right)\right|} \times I R_{+}^{\sum_{k=1}^{K}\left|J\left(E_{k}\right)\right|}$, such that (i) $\left(x_{1}^{i}, x^{i}(1), \ldots, x^{i}(\Xi), y^{i}\right) \in \arg \max u^{i}\left(x_{1}, x(1), \ldots, x(\Xi)\right)$, subject to constraints eqs (1) and ( 2), given $F[i ; \mathbf{I}]$; and (ii) $\sum_{i \in \mathbf{I}}\left(x_{1}^{i}-\omega_{1}^{i}+\sum_{E_{k} \in F[i ; \mathbf{I}]} \sum_{j\left(E_{k}\right) \in J\left(E_{k}\right)} g_{j\left(E_{k}\right)}\left(y_{j\left(E_{k}\right)}^{i}\right)\right)=0$, $\sum_{i \in \mathbf{I}}\left(x^{i}(\xi)-\omega^{i}(\xi)\right)=0$, for all $\xi \in \mathbf{\Xi}$, and $\sum_{i \in S_{k}} y_{j\left(E_{k}\right)}^{i}=0, \forall j\left(E_{k}\right) \in J\left(E_{k}\right), \forall E_{k} \in F(\mathbf{I})$.

\section{Theorem 1.}

Assume A1, A2, and A4. Then, an equilibrium exists for our economy with multiple exchange memberships and cross-listings. 


\section{Proof of Theorem 1:}

Let us consider a generalized game in which players maximize payoffs in truncated compact sets. The players are the traders and one auctioneer who chooses the prices for the securities. ${ }^{6}$ Let $W_{1}=\sum_{i \in \mathbf{I}} \omega_{1}^{i}$ denote the aggregate endowment of date 1 . Let $\bar{Y}=2 \max _{j\left(E_{k}\right) \in \mathbf{J}\left(E_{k}\right), E_{k} \in F[\mathbf{I}]}\left\{\left|g_{j\left(E_{k}\right)}^{-1}\left(W_{1}\right)\right|\right\}, \bar{W}=\max \left\{\sum_{i \in \mathbf{I}} \omega_{1}^{i}, \sum_{i \in \mathbf{I}} w^{i}(1)\right.$, $\left.\ldots, \sum_{i \in \mathbf{I}} w^{i}(\Xi)\right\}$, and $n \in I N$ and consider the closed cube: $C^{i}=[0, n] \times[0,2 \bar{W}]^{\Xi} \times[-\bar{Y}, \bar{Y}]^{\sum_{\left.E_{k} \in F[i ;]\right]}\left|J\left(E_{k}\right)\right|}$. We make $C^{i}$ large in order to guarantee that an equilibrium of the generalized game is an interior point. Each trader chooses a vector $\left(x_{1}^{i}, x^{i}(1), \ldots, x^{i}(\Xi), y^{i}\right)$ on $C^{i}$, subject to constraints eqs (1) and (2), for a given vector of security prices. Now fix $m \in I N$. The auctioneer chooses security prices in $[0, m]^{\sum_{E_{k} \in F(\mathbb{I})}\left|J\left(E_{k}\right)\right|}$ in order to maximize

$$
\sum_{i \in \mathbf{I}}\left(x_{1}^{i}-\omega_{1}^{i}\right)+\sum_{i \in \mathbf{I}} \sum_{E_{k} \in F[i ; \mathbf{I}]} \sum_{j\left(E_{k}\right) \in J\left(E_{k}\right)} g_{j\left(E_{k}\right)}\left(y_{j\left(E_{k}\right)}^{i}\right)+\sum_{E_{k} \in F(\mathbf{I})} \sum_{j\left(E_{k}\right) \in J\left(E_{k}\right)} q_{j\left(E_{k}\right)} \sum_{i \in S_{k}} y_{j\left(E_{k}\right)}^{i} .
$$

This auctioneer's objective function consists of the aggregation of all traders' budget constraints. We choose this specific objective function because it allows us to prove market clearing.

We denote the generalized game by $G(n, m)$. This generalized game has an equilibrium since it satisfies all the assumptions of Debreu's (1952) theorem. In fact, the auctioneer's objective function eq. (3) is linear in the respective price variables and the choice set is compact. Traders' utilities are continuous and strictly quasiconcave according to (A2), and their choice variables $\left(x_{1}, x(1), \ldots, x(\Xi), y\right)$ belong to non-empty, convex, and compact sets. Moreover, for each security price vector chosen by the auctioneer, the traders' choice set has an interior point. For instance, the price for the good is one and good endowments are assumed to be strictly positive. This assures the lower hemi-continuity of the budget set.

Lemma: Let us assume (A1), (A2), and (A4), and let $\left(\bar{x}_{1}^{I}, \bar{x}^{I}(1), \ldots, \bar{x}^{I}(\Xi), \bar{y}^{I}, \bar{q}\right)$ be an equilibrium of the generalized game $G(n, m)$. If $\bar{x}_{1}^{i} \leq W_{1}$ for all $i \in \mathbf{I}$, then there exist $\bar{m}$, such that $\max _{E_{k} \in F[\mathbf{I}] j\left(E_{k}\right) \in J\left(E_{k}\right)} \bar{q}_{j\left(E_{k}\right)}<\bar{m}$.

\section{Proof}

Without loss of generality, fix a security $j\left(E_{k}\right) \in J\left(E_{k}\right)$ and consider a trader $i \in \mathbf{I}$ such that $E_{k} \in F[i ; \mathbf{I}]$. By Assumption (A4), we have that, given $\theta \in(0,1)$, there exists $\varrho\left(\theta, W_{1},(2 \bar{W}, \xi=1, \ldots, \Xi)\right)$ such that $u^{i}\left(W_{1},(2 \bar{W}, \xi=\right.$ $1, \ldots, \Xi))<u^{i}\left(W_{1}+\varrho\left(\theta, W_{1},(2 \bar{W}, \xi=1, \ldots, \Xi)\right),(2 \theta \bar{W}, \xi=1, \ldots, \Xi)\right)$.

If we consider $\theta \in(0,1)$ such that $2 \bar{W} \theta<0.7 \omega^{i}(\xi), \forall i \in \mathbf{I}, \xi \in \Xi$, we conclude that $u^{i}\left(W_{1},(2 \bar{W}, \xi=\right.$ $1, \ldots, \Xi))<u^{i}\left(\omega_{1}^{i}+\breve{\varrho}, 0.7 \omega^{i}(1), \ldots, 0.7 \omega^{i}(\Xi)\right)$, where $\breve{\varrho}=\varrho\left(W_{1},(2 \bar{W}, \xi=1, \ldots, \Xi)\right)+W_{1}-\omega_{1}^{i}$. Note that $\varrho$ only depends on the primitive parameters of the economy.

The monotonicity of the preferences and the fact that $\bar{x}_{1}^{i} \leq W_{1}$ (later, we will show that this condition is satisfied in equilibrium), for all $i \in \mathbf{I}$, implies $u^{i}\left(\omega_{1}^{i}+\breve{\varrho}, 0.7 \omega^{i}(1), \ldots, 0.7 \omega^{i}(\Xi)\right)>u^{i}\left(\bar{x}_{1}^{i}, \bar{x}^{i}(1), \ldots, \bar{x}^{i}(\Xi)\right)$. Now, let us consider a trading position $\breve{y}_{j\left(E_{k}\right)}<0$ such that $-a_{j}(\widetilde{\xi}) y_{j\left(E_{k}\right)}<0.3 \min _{i \in \mathbf{I}, \tilde{\xi} \in \Xi} \omega^{i}(\widetilde{\xi})$ and $\omega_{1}^{i}-g_{j\left(E_{k}\right)}\left(\breve{y}_{j\left(E_{k}\right)}\right) \geq 0$. With this trading position on security $j\left(E_{k}\right)$, the bundle $\left(\omega_{1}^{i}-\bar{q}_{j\left(E_{k}\right)} \breve{y}_{j\left(E_{k}\right)}-g_{j\left(E_{k}\right)}\left(\breve{y}_{j\left(E_{k}\right)}\right), 0.7 \omega^{i}(1), \ldots, 0.7 \omega^{i}(\Xi)\right)$ is admissible for trader $i$ and, therefore, we can write $u^{i}\left(\omega_{1}^{i}-\bar{q}_{j\left(E_{k}\right.} \breve{y}_{j\left(E_{k}\right)}-g_{j\left(E_{k}\right)}\left(\breve{y}_{j\left(E_{k}\right)}\right), 0.7 \omega^{i}(1), \ldots, 0.7 \omega^{i}(\Xi)\right) \leq$ $u^{i}\left(\bar{x}_{1}^{i}, \bar{x}^{i}(1), \ldots, \bar{x}^{i}(\Xi)\right)$. Thus, we get inequality $u^{i}\left(\omega_{1}^{i}+\breve{Q}, 0.7 \omega^{i}(1), \ldots, 0.7 \omega^{i}(\Xi)\right)>u^{i}\left(\omega_{1}^{i}-\bar{q}_{j\left(E_{k}\right)} \breve{y}_{j\left(E_{k}\right)}-\right.$ $\left.g_{j}\left(\breve{y}_{j\left(E_{k}\right)}\right), 0.7 \omega^{i}(1), \ldots, 0.7 \omega^{i}(\Xi)\right)$, which implies $\bar{q}_{j\left(E_{k}\right)}<\bar{m}_{j\left(E_{k}\right)}=\frac{\breve{\varrho}+g_{j\left(E_{k}\right)}\left(\breve{y}_{j\left(E_{k}\right)}\right)}{-\breve{y}_{j\left(E_{k}\right)}}$ (recall that $\left.\breve{y}_{j\left(E_{k}\right)}<0\right)$. Since there is a finite set of securities, we can set $\bar{m}>0$ such that $\bar{m}>\max _{E_{k} \in F[\mathbf{I}]} \max _{j\left(E_{k}\right) \in J\left(E_{k}\right)} \bar{m}_{j\left(E_{k}\right)}$.

Next, let $\bar{n}=2 W_{1}+\bar{m} \bar{Y}$. We will show that if $(n, m) \gg(\bar{n}, \bar{m})$, then the equilibrium of the generalized game $G(n, m)$, denoted by $\left(x_{1}^{I}, x^{I}(1), x^{I}(2), \ldots, x^{I}(\Xi), y^{I}\right)$, is an equilibrium of the economy.

First, let us show that there is no excess of demand in the market of the good at the first date. Suppose not, $\sum_{i \in I}\left(x_{1}^{i}-\omega_{1}^{i}\right)+\sum_{i \in I} \sum_{E_{k} \in F[i ; I]} \sum_{j\left(E_{k}\right) \in J\left(E_{k}\right)} g_{j\left(E_{k}\right)}\left(y_{j\left(E_{k}\right)}^{i}\right)>0$. But then the auctioneer would choose $q=0$, and this would contradict the aggregation of the traders' budget constraints of the first date,

$$
\sum_{i \in \mathbf{I}}\left(x_{1}^{i}-\omega_{1}^{i}\right)+\sum_{i \in \mathbf{I}} \sum_{E_{k} \in F[i ; \mathbf{I}]} \sum_{j\left(E_{k}\right) \in J\left(E_{k}\right)}\left(q_{j\left(E_{k}\right)} y_{j\left(E_{k}\right)}^{i}+g_{j\left(E_{k}\right)}\left(y_{j\left(E_{k}\right)}^{i}\right)\right) \leq 0 .
$$

Now, we show that there is no excess of demand in the security market, $\sum_{i \in S_{k}} y_{j\left(E_{k}\right)}^{i} \leq 0$, for all $E_{k} \in F(\mathbf{I})$. If for some security $j\left(E_{k}\right)$, it happens that $\sum_{i \in S_{k}} y_{j\left(E_{k}\right)}^{i}>0$, then the auctioneer would choose the maximum possible price for this security, $q_{j\left(E_{k}\right)}=m>\bar{m}$, which is in contradiction with our previous result that security prices are uniformly bounded by $\bar{m}$ (notice that no excess of demand in the commodity market implies $x_{1}^{i} \leq W_{1}$, for every $i \in \mathbf{I})$. 
It remains to be shown that there is no excess supply in the good and in the security markets. If there were excess supply in the market of the good, we must have at least one trader with his budget constraint with strict inequality, but this contradicts the strict monotonicity of preferences. Thus, we conclude that

$$
\sum_{i \in I}\left(x_{1}^{i}-\omega_{1}^{i}\right)+\sum_{i \in I} \sum_{E_{k} \in F[i ; I]} \sum_{j\left(E_{k}\right) \in J\left(E_{k}\right)} g_{j\left(E_{k}\right)}\left(y_{j\left(E_{k}\right)}^{i}\right)=0 .
$$

If there were excess supply in the market of the securities $\sum_{i \in S_{k}} y_{j\left(E_{k}\right)}^{i}<0$, the auctioneer would choose $q_{j\left(E_{k}\right)}=$ 0 , again a contradiction with the strict monotonicity of preferences. Actually, if $q_{j\left(E_{k}\right)}=0$, every trader would choose $y_{j\left(E_{k}\right)}^{i}=0$. Otherwise, the trader has to pay a trading cost and gets no income from the short sale.

The proof that there is no commodity excess supply or demand in each node $\xi$ of date 2 follows by similar arguments as above.

Finally, we show that the bundle allocations are optimal. Market clearing in each market implies that $x_{1}^{i}<n$ and $x^{i}(\xi)<2 \bar{W}$, for any, $i \in \mathbf{I}$ and $\xi=\ldots, \Xi$. Moreover, $\left|y_{j\left(E_{k}\right)}^{i}\right|<\bar{Y}$. If $\left|y_{j\left(E_{k}\right)}^{i}\right| \geq \bar{Y}$ for any $i$, any $j\left(E_{k}\right)$ and any $E_{k}$, then $g_{j\left(E_{k}\right)}\left(y_{j\left(E_{k}\right)}^{i}\right)>W_{1}$, which, in turn, would imply that market clearing at date 1 does not hold. Thus, for any $i,\left(x_{1}^{i}, x^{i}(1), \ldots, x^{i}(\Xi), y^{i}\right)$ belongs to the interior of $C^{i}$. Suppose that there exists another allocation $\left(\hat{x}_{1}^{i}, \hat{x}^{i}(1), \ldots, \hat{x}^{i}(\Xi), \hat{y}^{i}\right)$ that is budget feasible and such that $u^{i}\left(\hat{x}_{1}^{i}, \hat{x}^{i}(1), \ldots, \hat{x}^{i}(\Xi)\right)>u^{i}\left(x_{1}^{i}, x^{i}(1), \ldots, x^{i}(\Xi)\right)$. For $\lambda$ small enough, $\lambda\left(\hat{x}_{1}^{i}, \hat{x}^{i}(1), \ldots, \hat{x}^{i}(\Xi), \hat{y}^{i}\right)+(1-\lambda)\left(x_{1}^{i}, x^{i}(1), \ldots, x^{i}(\Xi), y^{i}\right)$ belongs to $C^{i}$, is budget feasible and, by strict quasi-concavity of the utility function, $u^{i}\left(\left(\lambda \hat{x}_{1}^{i}, \hat{x}^{i}(1), \ldots, \hat{x}^{i}(\Xi)\right)+(1-\lambda)\left(x_{1}^{i}, x^{i}(1), \ldots, x^{i}(\Xi)\right)\right)>$ $u^{i}\left(x_{1}^{i}, x^{i}(1), \ldots, x^{i}(\Xi)\right)$, which is a contraction with the optimality of $\left(x_{1}^{i}, x^{i}(1), \ldots, x^{i}(\Xi), y^{i}\right)$.

The rest of this subsection discusses the relationship of our paper with the literature on restricted participation. ${ }^{7}$ There are two branches in this literature, one with exogenous portfolio constraints and the other with endogenous portfolio constraints. The latter specifies restrictions by functions that depend not only on the portfolio, but also on the security and commodity prices - see Cass, Siconolfi, and Villanacci (2001) and Carosi, Gori, and Villanacci (2001). This literature considers smooth economies determined by smooth utility functions and restrictions, and relies on techniques of differential topology to prove existence and regularity of demand functions. To capture the current market microstructure of trading across exchanges, we introduce restrictions on whom to trade with: a trader can trade the securities available in his exchanges only with those traders that also belong to his exchanges.

Since in our setting the restrictions are given exogenously, we follow the other branch of the literature (the one with exogenous portfolio constraints), which applies fixed point techniques to prove existence of equilibrium. Angeloni and Cornet (2006) and Aouani and Cornet (2009), and Balasko, Cass, and Siconolfi (1990), and Cornet and Gopalan (2010), among others, have contributed to this literature, and consider restrictions given by spanning conditions on the set of admissible portfolios. For instance, Cornet and Gopalan (2010) considered a spanning condition on the set of admissible portfolios and imposed a "financial accessibility" assumption on the payoff of each date and state of nature. Our payoff at the first date, however, is of a different nature due to the transaction cost; more precisely, our payoff function is nonlinear in portfolios.

Notice that, even without a transaction cost, we could not apply the results of equilibrium existence in Balasko, Cass, and Siconolfi (1990) and Angeloni and Cornet (2006), or Aouani and Cornet (2009). The reason is that we want our model to accommodate the situation where a trader cannot transact securities that are not available in the exchanges where he is a member, and these restrictions do not satisfy the "financial survival" assumptions considered in those models. In particular, Angeloni and Cornet (2006) require that portfolio sets contain a neighborhood of zero, which does not necessarily hold in our case. In addition, Balasko, Cass, and Siconolfi (1990) and Aouani and Cornet (2009) require a non-redundancy type hypothesis to bound portfolio choice sets. We do not need to impose this kind of assumption since these sets are endogenously bounded in our model as a consequence of the transaction costs.

\subsection{Demand Differentiability}

\section{Proposition 1}

Assume A1, A2, and A3. Then, the individual demand $\left(x^{i}(q), y^{i}(q)\right)$ is a continuously differentiable function in prices $q$, for $q$ in the interior of $[0, \bar{m}]^{\sum_{E_{k} \in F(\mathbf{I})}\left|J\left(E_{k}\right)\right|}$, with $\bar{m}>0$.

\section{Proof of Proposition 1:}

TT

The first order necessary and sufficient conditions for an interior optimum of a trader $i^{\prime}$ s problem with some of his constraints being non-binding are: $D_{1} u^{i}-\tilde{\beta}_{1}=0 ; D_{\xi} u^{i}-\widetilde{\beta}(\xi)=0$ and $-x(\xi)+\omega^{i}(\xi)+A_{\xi}^{i} y=$ 
0 for $\xi=1, \ldots, \Xi ; \tilde{\beta}^{T} A^{i}-\tilde{\beta}_{1}\left[q+D_{y} g\right]=0$ (where $T$ refers to the transpose of a matrix and $g(y)=$ $\sum_{E_{k} \in F[i ; \mathbf{I}]} \sum_{j\left(E_{k}\right) \in J\left(E_{k}\right)} g_{j\left(E_{k}\right)}\left(y_{j\left(E_{k}\right)}\right)$; and $-x_{1}+\omega_{1}^{i}-q \cdot y-g(y)=0$. The shadow price vectors for the budget constraints at date 1 and node $\xi$ of date 2 are $\tilde{\beta}_{1}$ and $\tilde{\beta}(\xi)$, respectively. The columns of the return matrix $A^{i}=\left|\cdots A_{k} \cdots\right|$ are those $A_{k}$ with $E_{k} \in F[i, \mathbf{I}]$. Then, the element $A_{\tilde{\xi}}^{i}$ denotes the line $\xi$ of the return matrix $A^{i}$. The matrix of second order derivatives with respect to $\left(x_{1}, x(\xi), \tilde{\beta}(\xi), y, \tilde{\beta}_{1}\right)$, where $x(\xi)$ and $\tilde{\beta}(\xi)$ are generic elements of the corresponding $\Xi$-vector, is:

$$
\mathbf{J}=\left[\begin{array}{ccccc}
D_{1}^{2} u^{i} & 0 & 0 & 0 & -1 \\
0 & D_{\xi}^{2} u^{i} & (-1) & 0 & 0 \\
0 & (-1)^{T} & 0 & A^{i} & 0 \\
0 & 0 & A^{i T} & -\tilde{\beta}_{1} D_{y g}^{2} g & -q-D_{y} g \\
-1 & 0 & 0 & -q^{T}-D_{y} g^{T} & 0
\end{array}\right]
$$

It is easy to see that the matrix $\mathbf{J}$ is non-singular (i.e., invertible). For this, we need to show that if $\mathbf{J} z=0$, where $z=\left(\breve{x}_{1}, \breve{x}(\xi), \breve{\beta}(\xi), \breve{y}, \breve{\beta}_{1}\right)$, then $z=0$. So let $z$ be such that $\mathbf{J} z=0$. Then, $z^{T} \mathbf{J} z=0$, and using some of the equations of the system $\mathbf{J} z=0, z^{T} \mathbf{J} z=0$ reduces to $\left(D_{1}^{2} u^{i}\right)\left(\check{x}_{1}\right)^{2}+\check{x}(\widetilde{\xi})^{T}\left(D_{\tilde{\xi}}^{2} u^{i}\right) \breve{x}(\widetilde{\xi})-\check{y}^{T}\left(\tilde{\beta}_{1} D_{y}^{2} g\right) \breve{y}=0$. Notice that this last equality can be written as

$$
\left[\begin{array}{lll}
\check{x}_{1} & \check{x}(\xi)^{T} & \check{y}^{T}
\end{array}\right]\left[\begin{array}{ccc}
D_{1}^{2} u^{i} & 0 & 0 \\
0 & D_{\widetilde{\zeta}}^{2} u^{i} & 0 \\
0 & 0 & -\tilde{\beta}_{1} D_{y \delta}^{2} g
\end{array}\right]\left[\begin{array}{c}
\check{x}_{1} \\
\check{x}(\widetilde{\xi}) \\
\check{y}
\end{array}\right]=0
$$

which implies $\check{x}_{1}=0, \check{x}(\xi)=0$, and $\check{y}=0$ by negative definiteness of $D^{2} u^{i}$ and $-\tilde{\beta}_{1} D_{y}^{2} g .{ }^{8}$ Then, back to $\mathbf{J} z=0$, we obtain $\check{\beta}(\xi)=0$. Finally, again with $\mathbf{J} z=0, \breve{\beta}_{1}=0$. Therefore, by the implicit function theorem, we conclude that a trader's excess demand is a continuous differentiable function in security prices.

\section{Further Remarks}

This section addresses three important issues related to our baseline model. The first one discusses how the equilibrium with multiple exchanges differs from its counterpart in a setting with a centralized market. The second issue has to do with our assumption of convex transaction costs. Here we discuss the economic interpretation and explain the difficulties of obtaining demand differentiability and equilibrium existence if we consider instead a concave transaction cost function. Finally, we explain that our model can be seen as a particular case of a more general model with only one global exchange market and traders facing exogenous portfolio constraints. In addition, for this more general model, we further clarify our contribution to the literature on incomplete financial markets with portfolio constraints.

\subsection{An Example: Centralized Versus Decentralized Exchanges}

Let us consider a simple example with two cases for a simple economy with three traders and two securities. In the first case, there are two exchanges (one security per exchange), where only one of the traders can trade in both exchanges. In the second case, there is only one exchange (with two securities), which we call the centralized exchange. This example highlights the important role that trading costs have for the equilibrium and traders' welfare, and ultimately on the market microstructure. When trading costs are the same, traders are better off in the centralized exchange. However, when trading costs are sufficiently lower in the decentralized exchanges than in the centralized exchange, traders are better off in separate (overlapping) exchanges. Roughly speaking, the potential high trading costs of a centralized exchange offset the better risk sharing that this exchange offers when compared to the case of decentralized exchanges. This result suggests that factors that influence trading costs, such as the search costs, valuation costs, inventory costs, information processing technology, computing capacity, trade execution speed, and clearing, to name a few, may have an impact on traders' welfare and ultimately on the stability of the exchange structure. ${ }^{9}$

Example: There are two dates, three states of nature at the second date, three traders $(i=1,2,3)$, and two possible exchange structures: (i) two decentralized overlapping exchanges, $\left\{E_{1}^{d}, E_{2}^{d}\right\}=\left\{\left(S_{1}^{d}, A_{1}^{d}\right),\left(S_{2}^{d}, A_{2}^{d}\right)\right\}$ with $S_{1}^{d}=\{1,3\}$ and $S_{2}^{d}=\{2,3\}$, and (ii) a centralized exchange $E^{c}=\left(S^{c}, A^{c}\right)$ with $S^{c}=\{1,2,3\}$. Each decentralized exchange has only one security. 
For the case of decentralized exchanges, we use the following returns: $A_{1}^{d}=\left[\begin{array}{lll}0 & 1 & 0\end{array}\right]^{\prime}$ if the exchange is $E_{1}^{d}$ and $A_{2}^{d}=\left[\begin{array}{lll}1 & 0 & 1\end{array}\right]^{\prime}$ if the exchange is $E_{2}^{d}$, where ' denotes the transpose of the matrix. For the case of a centralized exchange, we consider the same two securities, namely,

$$
A^{c}=\left[\begin{array}{ll}
0 & 1 \\
1 & 0 \\
0 & 1
\end{array}\right]
$$

For both the decentralized and centralized exchange structures, we consider the same functional form of the trading cost: $g_{1}(y)=\gamma_{1} y^{2}$ (for security 1 with returns $\left.\left[\begin{array}{lll}0 & 1 & 0\end{array}\right]^{\prime}\right)$ and $g_{2}(y)=\gamma_{2} y^{2}$ (for security 2 with returns [ 10 $\left.1]^{\prime}\right)$. However, the value of $\gamma_{1}$ and $\gamma_{2}$ might differ between exchange structures.

The corresponding good endowments for traders 1,2 , and 3 are $\omega^{1}=(6,6,0,6), \omega^{2}=(8,6,6,0)$, and $\omega^{3}=$ $(4,6,6,6)$, respectively. The utility functions of traders $i=1,2,3$ are, respectively,

$$
\begin{aligned}
& u^{1}\left(x_{0}, x(1), x(2), x(3)\right)=x_{0}+2 x(2) \\
& u^{2}\left(x_{0}, x(1), x(2), x(3)\right)=x_{0}+2 x(3) \\
& u^{3}\left(x_{0}, x(1), x(2), x(3)\right)=x_{0}+x(1)
\end{aligned}
$$

In the appendix, we report the equilibrium consumption and trading quantities as a function of $\gamma_{1}$ and $\gamma_{2}$ for each exchange structure. Using those expressions, we find that, for the case with two decentralized overlapping exchanges, the corresponding indirect utilities of traders 1,2 , and 3 are, respectively, $u^{1}=6+\left(1 /\left(4 \gamma_{1}\right)\right), u^{2}=8+$ $\left(1 /\left(16 \gamma_{2}\right)\right)$, and $u^{3}=10+\left(1 /\left(4 \gamma_{1}\right)\right)+\left(1 /\left(16 \gamma_{2}\right)\right)$. For the case with a unique exchange, the corresponding indirect utilities of traders 1,2 , and 3 are, respectively, $u^{1}=6+\left(4 /\left(9 \gamma_{1}\right)\right)+\left(1 /\left(4 \gamma_{2}\right)\right), u^{2}=8+\left(1 /\left(9 \gamma_{1}\right)\right)+\left(1 /\left(4 \gamma_{2}\right)\right)$, and $u^{3}=10+\left(1 /\left(9 \gamma_{1}\right)\right)$.

From the above expressions, we find that, if the three traders belong to a single exchange with $\gamma_{1}=\gamma_{2}=1$, then their respective utilities are lower than if they were in separate exchanges with $S_{1}=\{1,3\}$ and $S_{2}=\{2,3\}$, and $\gamma_{1}=0.3$ and $\gamma_{2}=0.1$.

\subsection{Convex Transaction Costs}

In real life, traders face several types of transaction costs. These include taxes, fees paid to the exchange where a trade is executed, and other types of costs, perhaps more indirect, but also economically important, that have to do with those frictions that traders face to execute trades. For example, traders may incur search costs, security valuation costs, inventory costs, and other costs related to some possible form of asymmetric information. Whereas transaction fees paid to the exchange are rather linear, and in some cases concave, other types of costs, such as the ones mentioned before, are usually convex.

The economic intuition behind convex search costs is simple: the larger the quantity a trader wants to buy/sell, the more difficult it is to find the security in case of a security purchase or buyer in case of a security sale. This type of friction has been coined as "market illiquidity" in the literature of search theory applied to financial markets (see, for instance, Lagos, Rocheteau, and Wright 2017). Traditional general equilibrium models have ignored such frictions. To some extent, we can incorporate these indirect costs into our model with a (proxy) transaction cost function that is convex in the amount of the security transacted.

The convexity argument also applies in terms of security valuation costs. Think for instance of a mortgagebacked security (MBS). This financial product consists of a pool of mortgages of possibly different types (e.g., different maturities, loan-to-value ratios, prepayment conditions and geographies, to name a few) that secure the MBS payments. Roughly speaking, the larger the amount of the MBS that a trader wants to buy, the more due diligence the trader has to do in order to properly understand and value the individual mortgages that back the MBS.

These are just some examples that motivate our assumption of convex transaction costs.

Finally, it is worth noting that we are considering a competitive framework, so the convexity of the transaction cost does not have to do with the price impact that traders may have in an environment with market power.

The rest of this subsection discusses the technical difficulties and potential solutions that one may face when dealing with other transaction costs that are concave, such as those paid by the traders to their respective exchanges. ${ }^{10}$ First, we cannot guarantee the differentiability of the trader's demand function using the approach of Geanakoplos and Polemarchakis (1986) when transaction costs are concave. This is because we need to have 
the matrix of second order derivatives with respect to $\left(x_{1}, x(\xi), \tilde{\beta}(\xi), y, \tilde{\beta}_{1}\right)$ invertible (see the proof of Proposition 1). But with a concave utility function (negative definiteness of $D^{2} u^{i}$ ), the transaction cost function needs to be convex (i.e., $\tilde{\beta}_{1} D_{y}^{2} g$ needs to be positive definite).

Second, Debreu (1952) fixed point theorem, used in our equilibrium existence proof, fails with concave transaction costs due to the non-convexity of the trader's budget set. Our existence result could be recovered if we were to consider instead a large economy with a continuum of traders. ${ }^{11}$ This approach has been used by Araujo and Pascoa (2002, Lemma 2) in a general financial equilibrium economy with incomplete markets and non-convex consumer budget sets.

\subsection{A More General Model with a Single Financial Market}

One could argue that the restuls found here could be also obtained under an alternative specification using the standard model of restricted participation with a single market. The idea would be to assign different labels ("colors") to securities with the same return structure that are however traded in different exchanges. In this section, we show that such an approach would face the same new technical problems (collinearity problem, lower semi-continuity, etc.) that we are facing with our formulation. Specifically, we argue that we can interpret our baseline model with multiple exchanges, cross-listings, and multiple exchange memberships as a particular case of a more general model with only one global exchange market and traders facing exogenous portfolio constraints. In addition, we expand our discussion on our contribution to the literature on incomplete financial markets with portfolio constraints.

Let us consider the same ingredients (uncertainty, trader's primitives, and security payoffs) as in the baseline model in Section 2.1. Because now there is a single global market, we find it convenient to denote the financial return structure by the payoff matrix $A=\left[a_{j}(\xi)\right]_{\Xi \times \mathrm{J}}$. Our notation for a portfolio is now specific to the whole set of securities $\mathbf{J}$, that is, a portfolio $y=\left(y_{j}\right)_{j \in J} \in I R^{J}$ specifies the quantity of each security. In addition, we also consider transaction costs, specified by a function $g_{j}: I R \rightarrow I R_{+}$and satisfying Assumption A1.

Importantly, we allow for repetition of some columns of the payoff matrix $A$. Securities in $A$ with the same return structure can be distinguished by the set of traders that are allowed to trade those securities and/or by their corresponding transaction costs. We specify the set of traders allowed to trade a given security using restrictions on their trading constraints. In particular, we assume that traders face portfolio constraints, i.e., each trader chooses a portfolio in $Y^{i} \subset I R^{J}$, which is the set of portfolios that trader $i$ is allowed to choose. We require the following assumptions on $Y^{i}$.

(A5) For every trader $i \in \mathbf{I}, Y^{i}$ is a closed and convex set with $0 \in Y^{i}$.

Let us now rewrite the trader's budget constraints for this new setting. Given security prices $q \in I R_{+}^{J}$, the trader $i^{\prime}$ s budget constraint at date 1 is

$$
x_{1}-\omega_{1}^{i}+\sum_{j \in \mathbf{J}}\left(q_{j} y_{j}+g_{j}\left(y_{j}\right)\right) \leq 0 .
$$

Trader $i$ 's budget constraint at date 2 and node $\xi \in \Xi$ is such that his consumption, net of his good endowment, is bounded by the returns on his security positions, i.e.,

$$
x(\xi)-\omega^{i}(\xi) \leq \sum_{j \in \mathbf{J}} a_{j}(\xi) y_{j}
$$

An equilibrium then consists of a system $\left(x_{1}^{I}, x^{I}(1), \ldots, x^{I}(\Xi), y^{I}, q\right) \in I R^{(1+\Xi) I} \times I R^{I I} \times I R^{I}$, such that, (i) $\left(x_{1}^{i}, x^{i}(1)\right.$, $\left.\ldots, x^{i}(\Xi), y^{i}\right) \in \arg \max u^{i}\left(x_{1}, x(1), \ldots, x(\Xi)\right)$, subject to portfolio constraints $y^{i} \in Y^{i}$ and to budget constraints eqs (4) and (5), and (ii) $\sum_{i \in \mathbf{I}}\left(x_{1}^{i}-\omega_{1}^{i}+\sum_{j \in \mathbf{J}} g_{j}\left(y_{j}^{i}\right)\right)=0, \sum_{i \in \mathbf{I}}\left(x^{i}(\xi)-\omega^{i}(\xi)\right)=0$, for all $\xi \in \mathbf{\Xi}$, and $\sum_{i \in \mathbf{I}} y_{j}^{i}=0$, $\forall j \in \mathbf{J}$. An equilibrium exists for this alternative economy, as we claim next.

\section{Theorem 2.}

Assume A1, A2, A4, and A5. Then, an equilibrium exists for the financial economy.

The proof of Theorem 2 follows the same steps of Theorem 1 and, therefore, we omit it. Next, we explain that the specificities of our general model require a different approach than the ones used in the literature of exogenous portfolio constraints to show equilibrium existence.

In the general model introduced above, the exogenous participation restrictions of a trader $i$ is defined by a set $Y^{i} \subseteq I R^{J}$, with $Y^{i}$ being a closed, convex set which contains zero. This framework includes linear equality 
and inequality constraints as particular cases and, therefore, it includes the case of linear subspaces of the portfolio space. However, the presence of transaction costs in our model makes it different from other models in the literature with exogenous constraints. Indeed, transaction costs play a key role in the proof of equilibrium existence as well as in the welfare properties and structure of exchanges, as we show in our example in Section 4.1.

An essential step in the proof of equilibrium existence is to find a non-empty, convex and compact choice set of portfolios. Assumption A5 guarantees that the portfolio choice set is non-empty, convex and closed. The subtlety in this proof is finding upper and lower bounds to guarantee that every trader's portfolio choice set is compact. In the incomplete financial markets without trading restrictions literature, this is obtained by assuming a payoff matrix with full rank; this is made without loss of generality since redundant securities can be removed without changing the equilibria consumption set. However, this is no longer true in a setting with individual trading constraints. There are different methods to overcome this difficulty. For instance, a wellknown technique in the literature consists of reducing the original financial economy (the financial structure or the set of admissible portfolio allocations) and keeping the same consumption equilibria (see Aouani and Cornet 2011 for a more detailed discussion). In our model, it is precisely the transaction cost that allows us to find endogenous bounds for the portfolio choice sets (see proof of Theorem 1).

Another difficulty to prove equilibrium existence is bounding security prices. Without portfolio constraints, the standard approach is to embed all prices in the simplex. With portfolio constraints, this approach puts at risk the lower semicontinuity of a trader's budget constraints when such a trader cannot transact certain securities. Aouani and Cornet $(2009,2011)$, and Angeloni and Cornet (2006) get rid of this problem by introducing "financial survival" assumptions, which allow all agents to short sell at least a minimum amount of each security. This approach is not compatible with our general model if we want it to have as a particular case our economy with multiple exchanges where some traders cannot trade the securities specific to an exchange they do not belong to. To overcome this difficulty, we consider instead Seghir and Torres-Martinez (2011) impatience assumption (our assumption A4), which allows us to find upper bounds for security prices.

Finally, we believe that our approach with multiple exchanges and cross-listings would be more natural and intuitive than the more general model introduced above if we were to extend our model to a framework where exchanges are endogenously formed as in Faias and Luque (2017).

\section{Conclusions and Directions for Future Research}

When traders can belong to several bourses and the same security can be traded in different exchanges, differentiability of the demand function with respect to prices may fail due to the "collinearity problem". However, when considering a richer model with convex transaction costs, we show that traders' demand functions are smooth even in a context with collinear securities among different exchanges. Also, we show that, in this framework, an equilibrium exists under a mild impatience assumption that is satisfied by many utility functions, does not depend on the representation of individuals' preferences, and does not require further assumptions concerning the portfolio sets.

It remains an open question how to endogenize the structure of security exchanges with multiple memberships. The results in the current paper make a step towards that goal by identifying conditions under which demand differentiability is achieved.

Also, we believe that the results presented in this paper can be useful to study other issues in the field of financial intermediation. We are thinking, for example, in a simple economy with two markets: a primary market where banks originate and buy mortgages from households, and a secondary market where these banks sell these mortgages to investors. This setting captures our main ingredients: banks belonging to more than one market, and the same assets (mortgages) transacted in the two markets; however, households and investors cannot transact with one other.

\section{Acknowledgements}

For their comments and suggestions, we would like to thank Bernard Cornet, Carlos Herves-Beloso, Juan Pablo Torres-Martinez, Yiannis Vailakis, the participants at the conferences of SAET (2013), EWGET (2013), and PET (2013), as well as the participants at the V Workshop on Equilibrium Analysis (2014), seminars at the U. Indiana, Purdue U., and the U. Wisconsin-Madison. Faias gratefully acknowledges that this work was partially supported by the Fundação para a Ciência e a Tecnologia (Portuguese Foundation for Science and Technology) through UID/MAT/00297/2013 (CMA). 


\section{Appendix}

\section{Example}

Let us denote securities with returns $\left[\begin{array}{lll}0 & 1 & 0\end{array}\right]^{\prime}$ and $\left[\begin{array}{lll}1 & 0 & 1\end{array}\right]^{\prime}$ by $j_{1}$ and $j_{2}$, respectively. For the case with two decentralized overlapping exchanges, we find the following equilibrium security prices and traders' consumption bundles:

$$
\begin{aligned}
\left(q_{j_{1}\left(E_{1}^{d}\right)}, q_{j_{2}\left(E_{2}^{d}\right)}\right) & =\left(1, \frac{3}{2}\right) \\
\left(x_{0}^{1}, x^{1}(1), x^{1}(2), x^{1}(3), y_{j_{1}\left(E_{1}^{d}\right)}^{1}\right) & =\left(6-\frac{3}{4 \gamma_{1}}, 6, \frac{1}{2 \gamma_{1}}, 6, \frac{1}{2 \gamma_{1}}\right) \\
\left(x_{0}^{2}, x^{2}(1), x^{2}(2), x^{2}(3), y_{j_{2}\left(E_{2}^{d}\right)}^{2}\right) & =\left(8-\frac{7}{16 \gamma_{2}}, 6+\frac{1}{4 \gamma_{2}}, 6, \frac{1}{4 \gamma_{2}}, \frac{1}{4 \gamma_{2}}\right) \\
\left(x_{0}^{3}, x^{3}(1), x^{3}(2), x^{3}(3), y_{j_{1}\left(E_{1}^{d}\right)}^{3}, y_{j_{2}\left(E_{2}^{d}\right)}^{3}\right) & =\left(4+\frac{1}{4 \gamma_{1}}+\frac{5}{16 \gamma_{2}}, 6-\frac{1}{4 \gamma_{2}}, 6-\frac{1}{2 \gamma_{1}}, 6-\frac{1}{4 \gamma_{2}},-\frac{1}{2 \gamma_{1}},-\frac{1}{4 \gamma_{2}}\right)
\end{aligned}
$$

Then, the corresponding indirect utilities of traders 1,2 , and 3 are, respectively $u^{1}=6+\left(1 /\left(4 \gamma_{1}\right)\right), u^{2}=8+$ $\left(1 /\left(16 \gamma_{2}\right)\right)$, and $u^{3}=10+\left(1 /\left(4 \gamma_{1}\right)\right)+\left(1 /\left(16 \gamma_{2}\right)\right)$.

For the case with a unique exchange, we find the following equilibrium security prices and traders' consumption bundles:

$$
\begin{aligned}
\left(q_{j_{1}\left(E^{c}\right)}, q_{j_{2}\left(E^{c}\right)}\right) & =\left(\frac{2}{3}, 1\right) \\
\left(x_{0}^{1}, x^{1}(1), x^{1}(2), x^{1}(3), y_{j_{1}\left(E^{c}\right)}^{1}, y_{j_{2}\left(E^{c}\right)}^{2}\right) & =\left(6+\frac{1}{4 \gamma_{2}}-\frac{8}{9 \gamma_{1}}, 6-\frac{1}{2 \gamma_{2}}, \frac{2}{3 \gamma_{1}}, 6-\frac{1}{2 \gamma_{2}}, \frac{2}{3 \gamma_{1}},-\frac{1}{2 \gamma_{2}}\right) \\
\left(x_{0}^{2}, x^{2}(1), x^{2}(2), x^{2}(3), y_{j_{1}\left(E^{c}\right)}^{2}, y_{j_{2}\left(E^{c}\right)}^{2}\right) & =\left(8+\frac{1}{9 \gamma_{1}}-\frac{3}{4 \gamma_{2}}, 6+\frac{1}{2 \gamma_{2}}, 6-\frac{1}{3 \gamma_{1}}, \frac{1}{2 \gamma_{2}},-\frac{1}{3 \gamma_{1}}, \frac{1}{2 \gamma_{2}}\right) \\
\left(x_{0}^{3}, x^{3}(1), x^{3}(2), x^{3}(3), y_{j_{1}\left(E^{c}\right)}^{3}, y_{j_{2}\left(E^{c}\right)}^{3}\right) & =\left(4+\frac{1}{9 \gamma_{1}}, 6,6-\frac{1}{3 \gamma_{1}}, 6,-\frac{1}{3 \gamma_{1}}, 0\right)
\end{aligned}
$$

Then, the corresponding indirect utilities of traders 1,2 , and 3 are, respectively, $u^{1}=6+\left(4 /\left(9 \gamma_{1}\right)\right)+\left(1 /\left(4 \gamma_{2}\right)\right)$, $u^{2}=8+\left(1 /\left(9 \gamma_{1}\right)\right)+\left(1 /\left(4 \gamma_{2}\right)\right)$, and $u^{3}=10+\left(1 /\left(9 \gamma_{1}\right)\right)$.

\section{Notes}

${ }^{1}$ See Malamud and Rostek (2017) for a different approach where a trader may have a price impact. In our paper we consider a large economy where trading in exchanges is Walrasian. Thus, when evaluating an exchange, a trader anticipates that his own participation has no price impact on security prices. Our context is naturally satisfied in exchanges with many traders.

${ }^{2}$ This assumption is also common in the literature of network formation. See, for example, Malamud and Rostek (2017)

${ }^{3}$ For example, we may consider an economy with three states of nature at date 2 and two bourses $S$ and $S^{\prime}$ with two securities and the following payoff matrices $A(S)=\{(1,0,0),(0,0,1)\}$ and $A\left(S^{\prime}\right)=\{(1,0,1),(0,1,0)\}$. These payoff vectors are clearly linearly dependent for a trader $i$ that belongs to the two exchanges and value consumption in every state of nature in period 2 .

${ }^{4}$ In our setting we add the transaction cost, which is a non-linear payment in each financial transaction in the first period. However, this is not the case in the model of Seghir and Torres-Martinez (2011). Moreover, they impose an assumption that requires that the portfolio choice set is bounded (their assumption A7). We do not need that assumption. Our existence proof incorporates these subtleties.

${ }^{5}$ For the sake of simplicity in the exposition of our proofs, we have decided to assume that transaction costs do not revert into the economy. That possibility could be incorporated into our model by considering a transfer system that distributes these resources back to the individuals of the economy according to some exogenous rule. See e.g., Markeprand (2008) and Préchac (1996).

${ }^{6}$ Since there is only one good, the price of the good is naturally 1 , so no additional auctioneers are needed here.

${ }^{7}$ See the seminal works of Balasko, Cass, and Siconolfi (1990), and Polemarchakis and Siconolfi $(1995,1997)$.

${ }^{8}$ This result would not be possible if we had assumed a concave transaction cost function (i.e., $g_{j\left(E_{k}\right)}$ concave in $y_{j\left(E_{k}\right)}$ ).

${ }^{9}$ See Glosten (1994) for a leading paper that analyzes the role of information processing technology on the stability and structure of trading institutions.

${ }^{10}$ With increased competition among exchanges, non-linear transaction fees paid to the exchanges are now more important. In many situations, these transaction fees are concave rather than convex, i.e., the larger the quantity, the lower the trading cost on average. For example, traders may pay a constant price per order, but some traders doing a high volume can sign up for a different offer involving a fixed part and a lower unit price. 


\begin{abstract}
${ }^{11}$ For this extension, notice that, following Hildenbrand (1974), most of the properties of our generalized game in the continuum setting would hold. Lyapounov's convexity theorem guarantees that the aggregate trader demand is convex-valued. To deal with the non-convexity of the trader 's budget set, one should extend the truncated generalized game to mixed strategies, and then show that an equilibrium exists for this extended generalized game by appealing to Debreu (1952) theorem. Since the auctioneer's new objective function depends only on the average of the traders' profile in each exchange, one could use Schmeidler (1973) to show that a degenerate equilibrium of the extended generalized game is an equilibrium of the original game (see p. 62, Theorem 3, in Hildenbrand 1974).
\end{abstract}

\title{
References
}

Angeloni, L., and B. Cornet. 2006. "Existence of Financial Equilibria in a Multi-period Stochastic Economy." Advances in Mathematical Economics 8: 933-955.

Aouani, Z., and B. Cornet. 2009. "Existence of Financial Equilibria with Restricted Participation." Journal of Mathematical Economics 45: $772-786$.

Aouani, Z., and B. Cornet. 2011. "Reduced Equivalent Form of a Financial Structure." Journal of Mathematical Economics 47: 318-327.

Araujo, A., and M. Pascoa. 2002. "Bankruptcy in a Model of Unsecured Claims." Economic Theory 20: 455-481.

Arrow, K., and C. Debreu. 1954. “Existence of an Equilibrium For a Competitive Economy." Econometrica 22: 265-289.

Balasko, Y., D. Cass, and P. Siconolfi. 1990. “The Structure of Financial Equilibrium with Exogenous Yields: The Case of Restricted Participation." Journal of Mathematical Economics 19: 195-216.

Carosi, L., M. Gori, and A. Villanacci. 2001. "Endogenous Restricted Participation in General Financial Equilibrium." Journal of Mathematical Economics 45: 787-806.

Cass, D., P. Siconolfi, and A. Villanacci. 2001. “Ceneric Regularity of Competitive Equilibria with Restricted Participation.” Journal of Mathematical Economics 36: 61-76.

Cornet, B., and R. Copalan. 2010. "Arbitrage and Equilibrium with Portfolio Constraints." Economic Theory 45: 227-252.

Debreu, G. 1952. "A Social Equilibrium Existence Theorem." Proceedings of the National Academy of Sciences 38: 886-893.

Faias, M., and ]. Luque. 2017. “Endogenous Formation of Security Exchanges." Economic Theory 64 (2): 331-355.

Ceanakoplos, J., and H. Polemarchakis. 1986. “Existence, Regularity, and Constrained Suboptimality of Competitive Allocations When the Asset Market Is Incomplete," Cowles Foundation Working Paper 652.

Glosten, L. 1994. “Is the Electronic Open Limit Order Book Inevitable?” Journal of Finance 49:1127-1161.

Hildenbrand, W. 1974. Core and Equilibrium in a Large Economy. Princeton: Princeton University Press.

Krishnamurti, C., J.M. Sequeira, and F. Fangjian. 2003. “Stock Exchange Covernance and Market Quality.” Journal of Banking and Finance 27: 1859-1878.

Lagos, R., G. Rocheteau, and R. Wright. 2017. “Liquidity: A New Monetarist Perspective.” Journal of Economic Literature 55: 371-440.

Malamud, S., and M. Rostek. 2017. “Decentralized Exchange.” American Economic Review, Forthcoming.

Markeprand, T. 2008. “On Financial Equilibrium with Intermediation Costs." Journal of Mathematical Economics 44:148-156.

Polemarchakis, H., and P. Siconolfi. 1995. “Ceneric Existence of Competitive Equilibria when the Asset Market is Incomplete: A Symmetric Argument." Economic Theory 6: 495-510.

Polemarchakis, H., and P. Siconolfi. 1997. "Ceneric Existence of Competitive Equilibria with Restricted Participation." Journal of Mathematical Economics 28: 289-311.

Préchac, C. 1996. “Existence of Equilibrium in Incomplete Markets with Intermediation Costs.” Journal of Mathematical Economics 25 (3):373-380.

Seghir, A., and J.P. Torres-Martinez. 2011. “On Equilibrium Existence with Endogenous Restricted Financial Participation.” Journal of Mathematical Economics 47: 37-42.

Schmeidler, D. 1973. “Equilibrium Points of Non-atomic Games.” Journal of Statistical Physics 7:295-301.

Treptow, F. 2006. The Economics of Demutualization: An Empirical Analysis of the Securities Exchange Industry. New York: Springer-Verlag, LLC. 